\title{
The Evaluation and Suggestion for the Development of Dried Tangerine Peels Industry a Case Study of Xinhui
}

\author{
Xinyao Wang ${ }^{1, *}$ \\ ${ }^{1}$ Guangdong Experimental School Guangzhou, Guangdong 510000, China
}

\begin{abstract}
Xinhui dried tangerine peels industry, which is represented by Xinhui Dried Tangerine Peels Village (XDV)", is the most representative among other dried tangerine peels. In the paper, the case study and qualitative method are used to analyze the current condition of the Xinhui dried tangerine peels industry, and to provide suggestions for the future development of the Xinhui dried tangerine peels industry. It turns out that the disadvantages of XDV are the relatively low popularity, a large number of small-sized competitors, and the small differentiation between XDV and other small producers, while the advantages of XDV are its deep culture and the high quality of XDV products. Therefore, the appropriate method for the future development of the XDV is the branding strategy since the disadvantages of the XDV can be solved by branding strategy, and the advantages are corresponding to the application of branding strategy. There are several steps to take to reach the success of the branding strategy in several aspects. The first step is to define a clear value proposition, which makes the direction of the development clear. Secondly, XDV should use a diversification strategy for the future development of its products. Also, with the improvement of products, promotion is necessary for the future development of XDV because a good promotion can attract more potential consumers and help XDV remain popular. Lastly, with high popularity, the price of the Xinhui dried tangerine peels from XDV should be lower since the sales have already made up for the cost of maintaining the "high-cost" channel. As a result, the development methods that this paper provides for the researchers and businessmen of Xinhui dried tangerine peels market would be helpful to apply and confirm in the future.
\end{abstract}

\section{Introduction}

Dried tangerine peels currently have a relatively large market. For Xinhui dried tangerine peels, the market for them is much larger than that for dried tangerine peels from other places in China. This is because Xinhui dried tangerine peels were brought by overseas Chinese from Wuyi district nearly one hundred and fifty years ago, and it has became famous since then. Xinhui dried tangerine peels, the representative of the dried tangerine peels, is the most famous dried tangerine peels among common citizens and customers because it represents the sense of home and yearn. The representative industry for Xinhui dried tangerine peels business is Xinhui Dried Tangerine Peels Village (XDV) located in Xinhui, and it entered the dried tangerine peels market in 2013. It was the dominant industry in Xinhui, and the main trading platform of Xinhui dried tangerine peels in China.

Many researchers have been studying the use of branding strategy in different fields of business, and it turns out that branding strategy is one of the most useful strategies for any kind of business. The branding strategy basically means creating a brand for a product or service, and the brand can, in turn, help promote the selling of the product or the service. A "brand" can show a good impression to the customers and attract potential customers in a short turn. In the long turn, a brand can represent a distinctive personality of the product or the service and even develop its own business culture. It is important for the future development of a kind of product or service because it provides a structure to use strong brands into other markets and combine strategy across several markets [1]. Branding strategy usually relates to the highest value of a corporation, which means the highest value of a company is created by the branding strategy [2]. It is an effective way to promote the products because people have the idea of using the products or services from a famous brand to show their high social status and prestige [3]. There are many steps that need to be fulfilled to obtain the success of the branding strategy. First, the brand requires promotion, which includes choosing a nice name for the brand. Second, the producer needs to choose an advertising strategy to support and communicate the name to the customers. Last but not least, it is critical to maintain the position of the brand by using upgrading and developing the new products in the same brand [4]. A Successful branding strategy can also increase

${ }^{*}$ Corresponding author: 3102005986@qq.com 
customer satisfaction [5]. All of these advantages of developing a brand can be brought to the product, and can finally create an emotional bond between the producers and the customers, and maintain the high profit of selling the products.

Accordingly, recent theoretical developments have revealed that branding strategy has both short-term and long-term benefits to a kind of product or service like XDV, which has already accumulated a certain amount of good reputation, in a giant market like the market of Xinhui dried tangerine peels industry. However, there is a further problem with the future development of XDV. Although most research has already analyzed the current condition of the market of Xinhui dried tangerine peels, as well as the function of the branding strategy in the success of a company, there are few types of research about the analysis of the current condition of the XDV and the future development of XDV. One way to overcome this problem is to apply the analysis and the branding strategy to the current condition of the XDV, and combine the branding strategy to different problems that XDV faces currently. To investigate the problem, the case study and qualitative method are used to examine the branding strategy and combine different aspects of the branding strategy to the problems that XDV faces, to effectively provide suggestions for the future development of XDV.

\section{DATA AND METHOD}

\subsection{Data}

The data collection of the case enterprises used in this paper began in 2019, and the investigation lasted for more than three months. Theory extraction, data collection, data analysis, data verification, and the final determination of the theoretical framework are included.

TABLE 1. Interview recording

\begin{tabular}{|c|c|c|c|c|}
\hline \multirow[t]{6}{*}{$\begin{array}{l}\text { The main overview of the } \\
\text { data collection } \\
\text { of the selected enterprises in } \\
\text { this article }\end{array}$} & $\begin{array}{c}\text { Interview } \\
\text { Enterprises }\end{array}$ & Interviewees & $\begin{array}{l}\text { Key words } \\
\text { of the } \\
\text { interview }\end{array}$ & Interview topic \\
\hline & $\begin{array}{c}\text { Jiangmen } \\
\text { Xinhui } \\
\text { Chenpi } \\
\text { village } \\
\text { market Co., } \\
\text { Ltd }\end{array}$ & Sales manager & $\begin{array}{l}\text { Difficulties, } \\
\text { solutions }\end{array}$ & $\begin{array}{l}\text { The problems DTV faces and their solutions to those } \\
\text { problems }\end{array}$ \\
\hline & $\begin{array}{c}\text { Jiangmen } \\
\text { Xinhui } \\
\text { Chenpi } \\
\text { village } \\
\text { market Co., } \\
\text { Ltd }\end{array}$ & $\begin{array}{l}\text { Individual } \\
\text { farmer }\end{array}$ & $\begin{array}{l}\text { The change } \\
\text { of price, } \\
\text { difficulties, } \\
\text { solutions, } \\
\text { help from } \\
\text { the mother } \\
\text { company }\end{array}$ & $\begin{array}{l}\text { The current condition of the plantation of dried } \\
\text { tangerine peels }\end{array}$ \\
\hline & $\begin{array}{c}\text { Hengyi } \\
\text { Roast } \\
\text { Goose } \\
\text { Restaurant }\end{array}$ & Sales manager & $\begin{array}{c}\text { The reason } \\
\text { for buying } \\
\text { dried } \\
\text { tangerine } \\
\text { peels from } \\
\text { XDV, the } \\
\text { consideratio } \\
\mathrm{n} \text { from } \\
\text { different } \\
\text { perspectives }\end{array}$ & $\begin{array}{l}\text { The reason for purchasing dried tangerine peels from } \\
\qquad \text { XDV }\end{array}$ \\
\hline & $\begin{array}{c}\text { No } \\
\text { company } \\
\text { background }\end{array}$ & Visitor & $\begin{array}{l}\text { Reason of } \\
\text { coming to } \\
\text { XDV, the } \\
\text { characteristi } \\
\text { cs of XDV } \\
\text { that attract } \\
\text { them }\end{array}$ & Impression about XDV \\
\hline & $\begin{array}{c}\text { Jiangmen } \\
\text { Xinhui } \\
\text { Chenpi } \\
\text { village } \\
\text { market Co., } \\
\text { Ltd }\end{array}$ & $\begin{array}{l}\text { Seller in the } \\
\text { XDV }\end{array}$ & $\begin{array}{c}\text { The } \\
\text { impression } \\
\text { of XDV, } \\
\text { difficulties, } \\
\text { solutions, } \\
\text { help from } \\
\text { XDV, } \\
\text { feeling } \\
\text { about the } \\
\text { corporation }\end{array}$ & The feeling of doing business in XDV \\
\hline
\end{tabular}

\subsection{Method}

A case study is a type of research method that is commonly used in social science. It includes a deep and detailed investigation of a specific case. It is properly defined as "an intensive study of a single unit with an aim to generalize across a larger set of units" [6]. It can 
demonstrate the value of the local branding process, which shows the importance of the local community when being involved in the development of the brand [7]. Case study can show a clear connection between each problem that the case shows [8]. Additionally, it can help produce a clear theory from specific research by combining the research features with the case it uses [9]. Case study is also capable of creating a strong description and deep understanding of the social content, which is relevant to the case that it focuses, meaning that the case can be analysed from a more comprehensive angle and results from different perspectives can be developed [10]. Last but not least, case study can reveal more problems of a big system from the analysis of a small case. These problems can be generalized to the big system because the minimal model of the case can provide with a deeper observation about the system [11].

There are several reasons for choosing XDV as the case for the evaluation of the future development of the Xinhui dried tangerine peels industry. First of all, XDV is the only one large-scale company in the Xinhui dried tangerine peels industry, which means it can basically represent the manipulation of the Xinhui dried tangerine peels industry. Secondly, it has a great contribution to the commercialization of the Xinhui Tangerine Peel Industry. It sets the standard for the quality of Xinhui dried tangerine peels, based on products' ages and ways of storing, and it creates a trading platform for individual Xinhui dried tangerine producers and buyers to trade in a fair and safe way. It also produces its own Xinhui dried tangerine by the corporation with farmers Xinhui dried tangerine peels from twelve areas that plant high-quality Xinhui dried tangerine peels. Lastly, it meets many difficulties, and some of them are tough and remain unsolved. It does meet many difficulties, like the large population of tourists and the difficulties of holding a large number of Xinhui dried tangerine peels. However, although it solves these problems with the help from the government, corporation with other industry and the upgrading of its storing technology, it does have some other problems, like the frequent appearance of the sellers of fake Xinhui dried tangerine peels and the decreasing reputation of the XDV. These problems that XDV faces are the typical problems that most cultural industry faces. As a result, it will be valuable for the producers to solve these problems. All of these statements show the importance of using a case study to analyze a particular type of industry and the reasons for using XDV as the case of analyzing the Xinhui dried tangerine peels industry.

\section{RESULT}

\subsection{Results From The Survey About Xinhui Dried Tangerine Peels Market}

Define abbreviations and acronyms the first time they are used in the text, even after they have been defined in the abstract. Abbreviations such as IEEE, SI, MKS, CGS, sc, $\mathrm{dc}$, and rms do not have to be defined. Do not use abbreviations in the title or heads unless they are unavoidable.

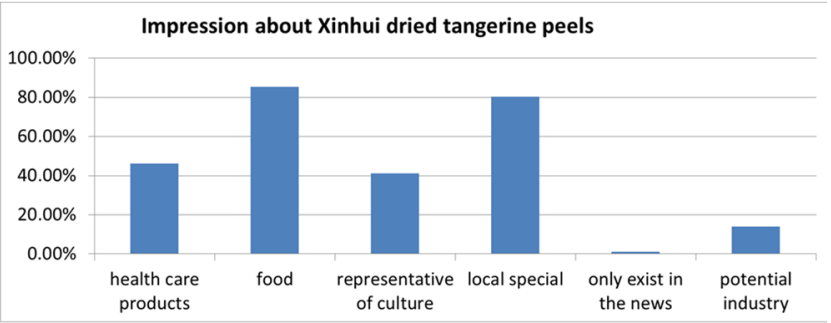

Figure 1. Impression about Xinhui dried tangerine peels

As seen in Figure 1, the impression on the Xinhui dried tangerine peels is that people mostly regard Xinhui dried tangerine as a kind of food or local specialty. This result shows that Xinhui dried tangerine peels have both practical function and memorial function to the customers. This is a clear advantage of creating a brand to promote the industry by using branding strategy because the producers can develop more kinds of products based on different needs of the consumers, and create a bond between the consumers and the producers themselves with the memorial function of the Xinhui dried tangerine peels.

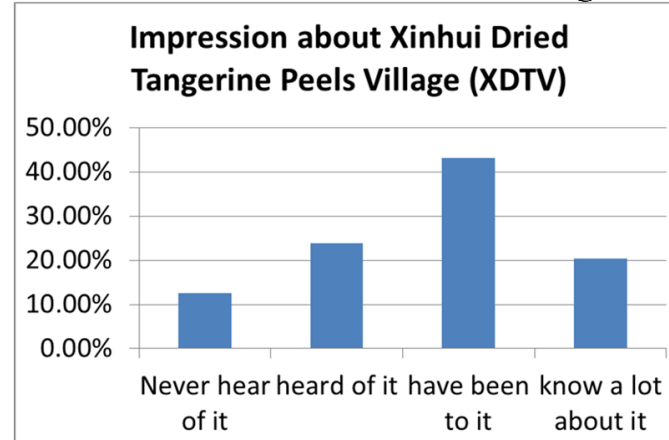

Figure 2. Impression about Xinhui Dried Tangerine Peels Village (XDTV)

Data source: from the survey by the WeChat questionnaire

It is schematically shown in Figure 2 that XDV has already accumulated a certain amount of reputation within the range of consumers from the Wuyi district, which is about the tenth of the Guangdong province. This is another advantage of using a branding strategy to promote the selling of the Xinhui dried tangerine peels because it demonstrates its high quality to the consumers who have an innate emotional bond with Xinhui dried tangerine peels. Obviously, a good reputation can better help strengthen the success of the branding strategy of XDV in the Xinhui dried tangerine peels industry.

\subsection{Disadvantages of XDV}

The result demonstrates three disadvantages of the XDV. First, the popularity of XDV is not large enough to create bonds or brands between consumers naturally because most people regard the dried tangerine peels as a kind of food or local special, which leads to an easy substitution of other products or other local specialties, like Hengyi Roast Goose. The purpose of purchasing Xinhui dried tangerine peels is also met by other products. Secondly, according to the interview with the sales manager, it reveals that there are too many small-sizeds competitors in the market who counterfeit their products as "Xinhui dried tangerine peels", with a lower price than that of the 
Xinhui dried tangerine peels from XDV, because there is no patent or restriction to the new producers who also sell "Xinhui dried tangerine peels." XDV has to maintain a relatively high price to cover the cost of manipulating the XDV, while the sellers of the fake "Xinhui dried tangerine peels" can lower the price to a greater extent. Therefore, it is hard for XDV to compete with these producers because generally, consumers would like to purchase Xinhui dried tangerine peels at a low price. This situation leads to the fact that small market share that can be taken by XDV. Last but not least, the differentiation between XDV and other producers is small. According to the interview, most citizens outside Xinhui have no idea of what XDV is, and they can not distinguish the Xinhui dried tangerine peels in the online shop, where they have interaction with Xinhui dried tangerine peels in common. Therefore, to some extent, it prohibits the promotion of XDV because most citizens do not realize the importance of buying Xinhui dried tangerine peels from the online or offline shop of XDV due to their lack of the understanding.

\subsection{Benefits of Branding Strategy Can Do for Thee Development of XDV}

In this case, the use of the branding strategy is pretty important. To begin with, branding strategy can help the promotion of the XDV, which creates a higher sale of the Xinhui dried tangerine peels. Since XDV has already applied for a patent of Xinhui dried tangerine peels, the involvement of the government is inevitable. The strong influence of the promotion from the government is crucial to the creation of the brand of XDV because it can help relieve the pressure of lowering sales while laying the foundation for the future development of the brand. Additionally, the money it creates can be used to invest in more advertisements. The advertisement is important to the success of the branding strategy because it has the potential to help increase the good reputation and a sense of fashion. This leads to the third point, which is the increase in consumer satisfaction. Consumer satisfaction includes two aspects. The first aspect is consumer loyalty to the product or the service. Consumers who regard the Xinhui dried tangerine peels from XDV as a kind of fashion will purchase the products of Xinhui dried tangerine peels in large amounts, therefore creates more sales in the future. The second aspect is that it provides more chances for the producers to try more products. Because of the confidence provided by loyal consumers, the producers have more courage to try to develop more kinds of products, which brings the management of the company to a higher level. Therefore, all of these advantages of branding strategy can effectively help the future development of XDV.

\section{CONCLUSION}

As a result, there are several steps that need to be taken to obtain the success of the branding strategy.

To begin with, defining a clear value proposition for the XDV itself is the fundamental step that needs to be taken. The most appropriate value proposition for the
$\mathrm{XDV}$ is "the platform for knowing Xinhui Dried Tangerine Peels." The reason for this is that it includes both the trading platform for producers and a sense of museum for tourists to know about the Xinhui dried tangerine peels. In this case, it provides both the financial and emotional bonds between the XDV, the tourists and the producers. The value proposition as the platform for knowing Xinhui Dried Tangerine Peels also creates its profit formula, which is to make profits by selling the dried tangerine peels, the tickets for the tourists when visiting the DTV, and the rent paid by other producers of stores or restaurant in the area of XDV.

Furthermore, the diversification of the products and services is also important for the future development of the XDV. Most consumers, especially the younger consumers, still consider the Xinhui dried tangerine peels as a bag of dry and rough peels in a dirty and old sack. However, this kind of stereotype should be broken. Since XDV has already accumulated a great reputation among the local consumers, it should make the products produced by Xinhui dried tangerine peels more diverse, to make the products more acceptable by younger consumers. XDV should separate the products made of Xinhui dried tangerine peels in different groups and pack them with elaborate and nice-looking packs because most young consumers purchase the Xinhui dried tangerine peels as snacks or gifts to their friends. Therefore, XDV should make their package good-looking and easy-carry. Also, nowadays consumers visiting a place of interest not only want to purchase some memorials but also want to enrich their knowledge and broaden their horizon. Therefore, XDV should provide not only a trading platform for professional buyers and consumers to exchange their products in a fair and safe environment, but also a relaxing and easy-understanding environment for tourists to know more about the history of Xinhui dried tangerine peels. For instance, XDV can regularly organize exhibitions about the history, and the historical meaning and values of Xinhui dried tangerine peels to the tourists. With more kinds of products and services produced, the differentiation between the XDV and other small producers is clear to the consumers.

In addition, promotion is also required to improve the products and the services. Recently, the way of XDV promotion is basically the communication between the consumers and some promotions from the government and the newspaper. With a clear differentiation from those producers who merely sold Xinhui dried tangerine peels, XDV can use more social media and TV shows to promote the distinction of itself as a platform for both trade and culture. It can also attract more individual local farmers to increase the sales and corporate with other companies like restaurants and even TV programs to further improve the reputation among the country. What is more, it can also use its own website and Wechat official account to maintain the loyalty of the frequent consumers. In this way, the promotion of the XDV can better help maintain its market position and strengthen its value proposition.

Last but not least, with the improvement of both products and promotion, the price of Xinhui dried tangerine peels can be lower. Recently, although the price of raw material is relatively low, the cost of pricing 
strategy is high because XDV uses high prices with lowcost channel with a "push" strategy to sell its products. Nevertheless, with the improvement of both products and promotions, it is beneficial for XDV to change its pricing strategy to a "pull" strategy, which accompanies relatively low prices and high-cost channel. Since the reputation of XDV attracts more consumers, it should lower its price of Xinhui dried tangerine peels on the online shops where most people can notice Xinhui dried tangerine peels and decide whether to purchase those products based on the price. A low price can increase the competitiveness of $\mathrm{XDV}$ in the market of online shops of dried tangerine peels. Also, a high-cost channel can maintain the quality of the products delivered to the consumers. This cost is worthy because the consumers will consider the XDV as a reliable company and finally decide to trust the XDV. Therefore, XDV should lower the price of Xinhui dried tangerine peels with the use of a high-cost channel that definitely ensures the quality of the Xinhui dried tangerine peels. In this case, a low-pricing strategy can help create and maintain the loyalty of the consumers.

\section{References}

1. Douglas S P, Craig C S. Dynamics of international brand architecture: Overview and directions for future research[J]. DYNAMICS, 2013, 21: 916-932.

2. Hsu L, Fournier S, Srinivasan S. Brand architecture strategy and firm value: how leveraging, separating, and distancing the corporate brand affects risk and returns $[\mathrm{J}]$. Journal of the Academy of Marketing Science, 2016, 44(2): 261-280.

3. Cheung M L, Pires G, Rosenberger P J, et al. Driving consumer-brand engagement and co-creation by brand interactivity[J]. Marketing Intelligence \& Planning, 2020. 98-111

4. Choudhury M, Mishra B B, Mohanty P K. An Empirical Study of Branding Strategy at Dealer point for Selling of Car-a qualitative \& systematic Review of Literature $[\mathrm{J}]$. International Research Journal of Automotive Technology, 2019, 2(1): 1-26.

5. Sezgin E, Demiral G N. Airports as shopfronts of tourism destinations: awarded brand Singapore Changi versus Surviving Istanbul Ataturk[M]//Eurasian Business Perspectives. Springer, Cham, 2019: 355-369.

6. Carvalho R L, Andresen E, Barônio G J, et al. Is dung removal a good proxy for other dung beetle functions when monitoring for conservation? A case study from the Brazilian Amazon[J]. Ecological Indicators, 2020, 109: 105841.

7. Eugenio-Vela J S, Ginesta X, Kavaratzis M. The critical role of stakeholder engagement in a place branding strategy: A case study of the Empordà brand[J]. European Planning Studies, 2020, 28(7): 1393-1412.

8. Kafka B. The demon of writing: Powers and failures of paperwork[M]. Mit Press, 2020. 99-108

9. Butt A S, Ahmad A B. Strategies to mitigate knowledge hiding behavior: building theories from multiple case studies[J]. Management Decision, 2020. 29-37

10. Ketokivi M, Turkulainen V, Seppälä T, et al. Why locate manufacturing in a high-cost country? A case study of 35 production location decisions[J]. Journal of Operations Management, 2017, 49: 20-30.

11. Hancock D R, Algozzine B. Doing case study research: A practical guide for beginning researchers[M]. Teachers College Press, 2017. 39-43 